\title{
Probiotics and their use in the treatment of the acute diarrhea
}

\section{The Bacterial Intestinal Flora}

In the human gastrointestinal tract is present a dynamic microbial population said "microbiota gastrointestinal", constituted by a community of living microorganisms distributed in the metabolic niches of the digestive tract. The intestine is the natural habitat of an elevated number of microorganisms and the microbiota contains either autochthonous kinds, acquired by the birth, or transitory microorganisms of environmental origin. Intestine represents, in fact, one of the organs in greater contact with different exogenous agents (bacteria, virus, allergens), constituting the first defensive barrier towards pathogenic microorganisms that can attack the organism [1-3].

\section{Development of the Human Microflora Intestinal}

The colonization in the gastrointestinal tract has begins with the birth and continues with advancing of the age before forming a peculiar microflora for each individual. Immediately after the birth intestine begins to populate a series of microorganisms modifying themselves for effect of exogenous and endogenous factors. During the first 12-24 hours of extra-uterine life, there are anaerobes as Escherichia coli, Enterococci and Streptococcus to colonize. Subsequently, to the second-third day, the obliged anaerobes are established (Bifidobacteria and Lactobacilli). In the arch of ten days, the bacterial population expands and there are established kinds as Bacteroides and Clostridia. After the first six months of life, with the beginning of the period of weaning, the diversification of the diet and the introduction of solid foods, there is a further differentiation of the microorganisms; the flora is enriched, in fact, with kinds present also in adult age, particularly with Bacteroidetes and
Firmicutes. The initial colonization results to define the definitive bacterial flora in adult age; in fact, once constituted, it remains stable with exception of possible variations following different factors of several nature how, for example, a change of the alimentary habits or the beginning of pathologies [4-7].

\section{- The microbiota}

The human microbiota with specific functions includes at least $10^{14}$ bacteria (with a total weight of about $1,5 \mathrm{~kg}$ ), 2000 kinds with the predominance of anaerobic bacteria; it is dominated by five phyla bacterial:

- Firmicutes (Lactobacillus, Ruminococcus, Clostridium, Mycoplasma, and Eubacterium)

- Gram-positive Actinobacteria (between which the Bifidobacterium)

- Gram-negative Bacteroidetes

- Proteobacteria (Escherichia coli)

- Verrucomicrobia

These bacteria have a very active metabolism: they degrade the polysaccharides as the cellulose producing fatty acids and oligosaccharides, produce vitamins, hydrogen, methane, and sulfured products, intervene in the regulations at the level of the mucosal barrier.

\section{The Probiotics}

The WHO defines the probiotics as "microorganism living and vital, swallowed in definite quantities, exert a beneficent action over the state of health" [8-10]. A great number of scientific publications of the last years assert that the ability of some kinds of microorganisms of acting as beneficent agents for the organism is strictly tied to some requirements that are satisfied:
Merra $\mathrm{G}^{1 *}$, Capacci $\mathrm{A}^{2}$, Ferrigno $\mathrm{F}^{1}$, Di Renzo $\mathrm{L}^{3}$, Franceschi $F^{1}$ and

\section{De Lorenzo $\mathrm{A}^{3}$}

${ }^{1}$ Emergency, Anesthetic and Resuscitation Sciences Department, "A. Gemelli" General Hospital Foundation IRCCS, Catholic University of Sacred Heart, Rome, Italy

${ }^{2}$ Gastroenterological, EndocrineMetabolic, Renal and Urological Sciences Department, "A. Gemelli" General Hospital Foundation IRCCS, Catholic University of Sacred Heart, Rome, Italy

${ }^{3}$ Section of Clinical Nutrition and Nutrigenomic, Department of Biomedicine and Prevention, University of Rome Tor Vergata, Rome, Italy

*Author for correspondence:

giuseppe.merra@policlinicogemelli.it 
- the microbial kind must be chosen between those that are normally present in the gastrointestinal tract or must be of human and not pathogenic origin

- an adequate number of bacterial cells must be able to survive during the transit through the stomach and the small intestine and then they must be resistant to the acid, to the bile and to the pancreatic saps

- they must be able to colonize and stick to the epithelium, resisting to the peristaltic movements

- do not lose the own characteristics during the conservation

- to be able to produce antimicrobial substances (bacteriocins)

- to be able to inhibit the pathogenicity

- to be stable genetically

- to be clinically evaluated with dutiful documentation of the beneficent effects

The tolerability and the certainty of the probiotics at present are excellent because they are, for definition, not pathogenic. Still, in immunosuppressed or weakened patients, rare cases of local infection have been brought back or systemic that have had a favorable result with agreed therapies. The risk, in the administration of living microorganisms, is the transfer of microbial resistance acquired to other organisms. Till now this event has never been verified in the clinic. The disposable data are reassuring, but it is needed to underline that for probiotic, systematic studies value the certainty for a long term. The commonest probiotic used bacteria belong to the family of Streptococcus, Lactobacilli, and Bifidobacteria.

In the guidelines that define what are probiotics, adopted by the international companies and Ministry of Public Health, have been taken in consideration the correct identification of the bacterial stump, the real dose, the certainty and the evaluation of the efficaciousness.

The identification: there are so many people and bacteria are different between them; some also belong to the same "bacterial family".

The real dose is the quantity of living and vital bacterial cells to be given to an individual, in order that the probiotic properties have been expressed.
The certainty has been proved in vitro and in vivo and, the greatest part of the probiotic at present well-known are organisms included in the list GRAS (Generally Recognized As Safe) and, therefore, their utilization is considered sure.

The efficaciousness of a probiotic stump has been valued by in vivo tests, during which the volunteers have consumed the product under medical control and have submitted to specific tests.

\section{Mechanisms of action proposed for probiotics}

The mechanisms through which the probiotics exercise the own biological actions are not completely well-known and certainly, they are numerous. Between these it is the substance production to antibactericidal activity like the bacteriocin, the lactic acid, the peroxide of hydrogen and some protease, the competition at the level of the connection sites and for the nutritive substances with the pathogenic microorganisms, an effect on the mucous intestinal membrane, an action normalised on the intestinal permeability and, at last, the stimulation of the immune system, both intestinal (GALT) and systemic. The probiotic bacteria are able to produce active substances to inhibit the growth of bacteria belonging to kinds potentially pathogenic. Such substances, called bacteriocins, are of peptidic nature produced by Gram-positive bacteria, preferentially by lactic bacteria (lactobacilli and bifid bacteria) belonging to kinds different from the producer. The existence of competition between bacteria that normally colonize intestine and those potentially pathogenic represents a second action mechanism. The "good" bacteria stick the brush-border of the intestinal epithelial cells forestalling the attack and the penetration of harmful bacteria. The competition exists also for nutrients: the bacteria that normally constitute the intestinal microflora maintain the own ecologic niche consuming the nutrients supplied by the host. This relation forestalls the increase of availability of nutrients that might favor the development of pathogenic bacteria.

The intestinal bifidogenic flora is able to metabolize a considerable number of not digestible oligosaccharides (prebiotic). These compound are fibers that reach unchanged the colon resisting the enzymatic digestion and are 
able to stimulate selectively the proliferation or the activity of determinate bacterial kinds because they represent their selective metabolic substrate. The fermentation of these compound (fruit-oligosaccharides) from bifidobacteria and lactobacilli leads to the formation of short chain fat acids (acetate, propionate and butyrate), which act as elective energy substratum for the colonies, winged substances and organic compound, and as energy substratum for the organism (hydrogen, carbonic anhydride, lactate, pyruvate and succinate). The probiotic bacteria are able to promote immunity defenses of the host modulating the answer to the attack from the pathogenic and reducing the hypersensitiveness reactions. The oral administration of lactobacilli increases not specific resistance of the host and facilitates the exclusion of the pathogenic microorganism of the gastrointestinal tract.

Some lactobacilli stump able to stimulate cytokines production from the macrophages and to activate the phagocytosis in experiments in vivo performed on mice. The phagocytosis is responsible for the precocious activation of the immune response. The phagocytes give toxic substances (reactive kinds of oxygen and lytic enzymes) in several inflammatory reactions. The ability of the probiotics of modulating phagocytosis is nevertheless different in the healthy subjects compared to the allergic subjects: the healthy subjects react developing an immunostimulatory effect, while in the allergic patients there is a "down-regulation" of the inflammatory response. 


\section{References}

Guarner F. Enteric flora in health and disease. Digestion. 73, 5-12 (2006).

Fioramonti J, Theodorou V, Bueno L. Probiotics: what are they? What are their effects on gut physiology? Best Pract. Res. Clin. Gastroenterol. 17(5), 711-724 (2003).

Kau AL. Human nutrition, the gut microbiome, and the immune system. Nature. 474, 327-336 (2011).

Wu GD. Linking long-term dietary patterns with gut microbial enterotypes. Science. 33, 105-108 (2011).

Cani PD. Changes in gut microbiota control inflammation in obese mice through a mechanism involving GLP2 driven improvement of gut permeability. Gut. 58, 1091-1103 (2009).

Gabrielli M, D'Angelo G, Di Rienzo T, Scarpellini E, Ojetti V. Diagnosis of small intestinal bacterial overgrowth in the clinical practice. Eur. Rev. Med. Pharmacol. Sci. 17 Suppl 2, 30-35 (2013).

Purchiaroni F, Tortora A, Gabrielli
$\mathrm{M}$, et al. The role of intestinal microbiota and the immune system. Eur. Rev. Med. Pharmacol. Sci. 17(3), 323-333 (2013).

Gigante G, Tortora A, Ianiro G, et al. Role of gut microbiota in food tolerance and allergies. Dig. Dis. 29(6), 540-549 (2011).

Gasbarrini A, Lauritano EC, Gabrielli $\mathrm{M}$, et al. Small intestinal bacterial overgrowth: diagnosis and treatment. Dig. Dis. 25(3), 237-240 (2007).

https://www.who.int/foodsafety/fs_ management/en/probiotic_guidelines.pdf 